\title{
Issues Affecting Health Research Collaborations based on Cloud Computing
}

\author{
Robert Mugonza, Annabella H. Basaza-Ejiri
}

\begin{abstract}
Research in science and technology is multifaceted with data sharing as a crucial issue among others. The issues are prevalent in collaboration based setups with significant interest in public health and medical where failure to share data, derails improvements in health and policy that is dependent on research outcomes. With data collected, it is stored on conventional storage including researcher PCs, and external hard disks. Researchers often find it hard to share this corpus data and if implemented under systems, users are left unsure of the confidentiality, transparency and ultimately the reliability of these systems. Existing research environments have not observed these needs, hence affecting collaborations, including those based on the cloud. This study utilizes grounded theory in investigating these issues. We have identified these issues including Reliability in terms of Data privacy, confidentiality, and integrity; Data sharing; Organizational change and Innovation resistance. We report that Cloud opportunities and benefits are reasons for increased adoption and these issues as hindrances as well. We recommend a characteristic cloud environment that is reliable, managed and offers accessible data sharing, and inclusive Service Level Agreements as a means to provide for the mutual relationship required between researchers in developing and developed countries; and conclude that the identified issues if observed and recommendations implemented, healthier collaborations will be possible.
\end{abstract}

Index Terms-Cloud Computing, Collaborations, Health, Biomedical, Research.

\section{INTRODUCTION}

Research in Science and technology today presents opportunities for transformation of societies especially within health, agriculture, environment and so forth. And in the recent decade, there has been a fast development of Information technology (IT) solutions in the health and medical domains. This has led to a number of benefits including specific and complex medical applications, increased speed in data processing, hardware infrastructure among others, enabling existing research progress and provision of better health services[1].

More so, the medical and health domains continue to release a lot of data - BigData, for research and industry requires the application of advanced techniques and resources in regards to computational and storage capabilities. Pursuing efficient computing for health research collaboration is just recent as discussed by[2], [3] \&[4]. Cloud computing is known for providing scarce computing resources (computing power, memory, storage, etc.) as a service over the network [2]. The term is also includes both the applications delivered as

Robert Mugonza, Department of Computer Science, Mbarara University of Science and Technology, Mbarara, Uganda

Annabella H. Basaza-Ejiri, Department of Computing, St. Augustine International University, Kampala, Uganda services over the Internet and the hardware and systems software in the datacenters that provide those services. So, a cloud computing environment may simply refer to combinations of components (layers), and subcomponents required for a particular task as observed in the fig-1, allowing users from distant geographic locations to share computing resource including data.

Managing sharing of data and other resources within these domains is too complex in regards to confidentiality, the integral need for the data and underlying system, and variations in governing policies.

In health and medical research, implementing cloud computing technologies can effectively assist researchers to improve the quality of medical services[5] and the efficiency of operations[6], pervasive sharing of information, and optimize on service overheads[7]. More so, enterprise-wide data sharing can help service providers increase efficiency at relatively low cost. The concept can be applied in a variety of ways, including data storage and preventing data loss, maintaining patient information records, and authorized sharing of information, which can be done quickly and efficiently on the cloud. However, the adoption of such systems and modes of application in the health research collaboration is limited in the developing world. There is need to investigate further into issues affecting public health and biomedical research collaborations that are supported by cloud computing.

The objective of this study was to investigate issues affecting public health and biomedical research collaborations that utilize cloud computing as a base computing environment. We utilize existing literature and a field study to attempt understand these issues. We utilize the case of Mbarara University of Science and Technology (MUST) in Uganda, a developing country, particularly to situate the study. MUST is a research and academic community specially established within health and biomedical research. Funded by NIH, government of Uganda, The Netherlands NUFFIC, The Swedish SIDA, National Science Foundation(NSF) and many more others, MUST engages in research with collaborators from Europe, United States of America(USA), Asia and Africa as well with particular attention to the achieving the collaboration objectives and a fully-fledged Memorandum of Understanding as a binding document. The role of the university in these researches is as a research implementation partner or a study investigator.

A research study that involves MUST and other partners pursing a common research objective whose geographical location is outside Africa - South - North OR within Africa - South-South, is referred to as collaboration. Collaborations under focus include all the above forms. This participation specifically attached to both South - North and South-South, avails an opportunity to get involved on with 
data, including data collection, sharing, analysis and dissemination, hence the reason for selecting MUST as a base case.

This paper is organized as follows: Section-II provides some works related to health research collaborations based on cloud computing. Section III describes the methodological approach utilized, findings and attempt to discuss these findings respectively. Finally Section VI provides conclusions and an insight for future work including recommendations for a characteristic cloud environment that enables effective and efficient collaboration activities.

Layers

Resource Management at Each Layer

\section{Examples}

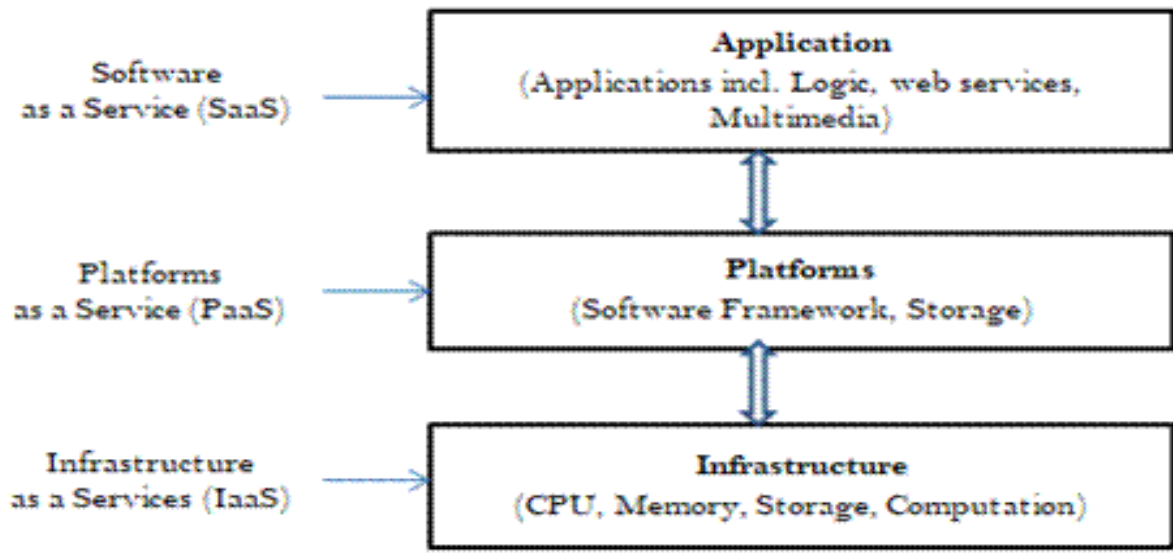

Google Apps like GMAIL \& Drive, DropBox, and OpenMRS etc.

MS Azure, Google AppEngine, ete.

Amazon EC2, Data Centre, GoGrid, etc.

Fig-1: Layered view of Cloud Computing,

\section{RELATED WORK}

Collaboration in the context of health research is not new. Health research can take on a number of forms including clinical trials of drugs and vaccines, Randomized trials, surveys, through the quantitative studies. It is argued by [8] and [9] that these emerge from mutual agreements and initiatives among collaborating parties onto defined common goals and shared intentions. Research collaboration in the context of developing and developed countries researchers, can be understood through perceived task divisions for the participating parties. In particular, research financing, design and analysis expertise for the research project is perceived for developed country researchers while locals (developing country researchers) are attached to research project data collection and many more forms of field[10]. A close observation of the context continues to identify developed country researchers as the ones with the idea, knowledge and skill[11], hence the unbalanced collaboration nature of health research.

Observed as a solution, the cloud may involve the provisioning of services that are encapsulated[12], with elastic access[13] to resources by developers and a user at a pay per use basis, providing a seamless view of infinite resources to users via virtualization[14] \& [15] as the enabling technology. With virtualization each user task is encapsulated into standard deployment objects, called virtual machines, offering flexibility due to the abstraction of the server hardware so that software stacks can be deployed and redeployed rather than over reliance on a specific physical server. Cloud based health collaborations have been realized also recently in the developing world ("the North") with developments of: - Seven Bridges Cancer and sharing on Medical Images of crossing the different hospitals' issues. And while design principles for the DataSHIELD are promising, data governance, trust, and data migration issues were not yet addressed; PACS Cloud Gateway to access PACS Cloud archive, is a private cloud funded by Chinese government to provides a high security level and without cloud provider's dependence. This solution challenges arise with cloud storage of medical images with unease presented by users and lack of trust regarding health practitioners' interpretation of the medical image, fault prescription[17], among others; Medical Image file Accessing System (MIFAS), a solution to exchanging, storing and sharing of medical images across different hospitals, is based on the support of the Hadoop and co-allocation mechanisms to establish a cloud environment enabling the sharing and exchange of the data. While with MIFAS its design objective was achieved with enhanced fault tolerance as presented in[18], presents that the cloud system is limited with regards to secure access to stored medical images. With the above implemented in developing countries ("South") by their collaborators, they assert that security is a major problem in the cloud.

Typically also, the cloud has been often used in a variety of ways including provision of free email services like that of Gmail and Yahoo mail, and mail.

However, fewer studies[19], [20] \& [21] discuss cloud computing adoption issues in the perspective of the actual users, and specifically within the health research collaboration domain, a number of issues have not been presented with a cloud user based assessment with special interest in users from the developing countries participating 
is such consortia supported by the cloud. We believe upon rigorous literature review that this is the first empirical paper studied on issues affecting health research collaboration powered by the cloud. This paper contributes evidently from a user perspective to Cloud computing literature, generate emerging concepts and attempting to articulate challenges for fast/slow adoption in a busy health research institution in the global south like Uganda.

\section{METHODOLOGY}

The methodology that is employed in this study is the grounded theory. This theory initially described as a way of generating theory from data by its proponents[22], it is an inductive qualitative based research approach postulating that the resulting theory is grounded in the data. This sociological and pragmatic approach has way been used since the $18^{\text {th }}$ century and has been adopted also in computing majorly in fields like Information Systems for example in Computer Aided Design of Software Engineering tools for investigating incremental and radical changes in systems Development[23] Computing Education for example in Computer Programming education [24, 25] in among others, and has been improved and its variants: Computational Grounded Theory[26]: grounded theory improvements using machine learning techniques too and others utilize somewhat a similar methodological style. Interestingly, this research adopts the classical grounded theory because of its view around new research areas including cloud computing application in health research collaboration in a somewhat busy research environment in Uganda. To achieve an initial understanding in the field prior to the data collection, a literature review was worth it, attaining clarity and depth of areas within Cloud computing, sufficient and does not violate the basic premise of the theory. Surveying a little literature on health research collaboration supported by cloud computing, returned very few results. However, many of these included those funded by developed countries governments including CGC (US), DataSHIELD (UK), PACS and MIFAS (China) among others with non from developing countries, a serious gap apparent in global south like sub Saharan Africa. In this study we attempt and fix the gap with empirical investigation into "what issues affect health research Collaborations that utilize cloud computing?" concentrating on a much busy research environment like Mbarara University of Science and Technology in Uganda.

\section{A. Research Sites}

Since previous research conducted on health research collaboration and cloud computing and how the later, is utilized in the perspective of South-South and North-South collaborations with in health related research studies is scarce, acquiring an overall understanding of the health research collaboration and Cloud Computing architectures along with the perceived benefits and drawbacks related to them was important. The paucity of prior research is intense more so within the context of Cloud computing paradigm adoption in developing countries research communities, a case for Uganda. It is because of this that the research site chosen for this study is Mbarara University of Science and Technology, a Ugandan research institution. Attempting to inform sampling and research participants, pieces of information regarding health research collaborative studies, both previous and current, gathered from the Institution's research and management office was utilized. A summary of profiles of these collaborative studies are presented in table-1.

\section{B. Data collection}

Utilizing an exploratory research method, the qualitative data was collected through face to face semi-structured interviews, supported by observations using the Glasserian grounded theory approach. An interview guide and a consent form were provided before beginning any interview to introduce the topic under discussion and to ensure that consent has been made to participate in the study. The structure utilized of the semi-structured interview and the development of the guide is informed by Barbara DiCicco- Bloom's article, the Qualitative Research interview[27]. And while all participants were asked the same questions as prepared in the interview guide, the exact wording and order / sequence were determined in the course of the interviews. An example interview guide is provided as an appendix 1. The interviews questions relate to different issues within Cloud computing in the health research collaboration. The selection of different professional interviewees encouraged us to design the interview guide with different questions focusing on their related area of interest and technologies in used. While constructing the questions for interview, it was kept in the mind that the interviewees who are technical should easily understand the main goal of the questions. To achieve this result we used information technology word instead of Cloud Computing while interviewing the non-technical people. Participants were categorized in various job roles as also indicated in the table below.

In Table-2, a total of 11 participants were selected using

TABLE-1: HEALTH COLLABORATIONS UTILISED FOR THE STUDY

\begin{tabular}{cccccc}
\hline \hline Study & Acronym of study & Health Domain Specific(TB/HIV) & $\begin{array}{c}\text { Status (On-going } \\
\text { /Completed) }\end{array}$ & No of employees & No of IT staff \\
\hline A & ADEPT & HIV/TB & On-going & 11 & 2 \\
B & WISEPILL & HIV & Completed & 6 & 1 \\
C & NCD & HIV & Completed & 7 & 2 \\
D & ISS-DATA & HIV/TB & On & 13 & 2 \\
E & CAMTECH & Other & On & 5 & 3 \\
F & ACHievA & Other & Completed & 5 & None \\
G & AIR & Other & On-going & 2 & None \\
\hline \hline
\end{tabular}


purposive sampling and later interviewed. The participants were requested to choose a more appropriate and conducive environment for the interview however, we ended up having the interviews in the office premises and in English to ensure that transcription does not raise any errors. The selection of the population for the interview is a critical and important part of the research methodology. I have selected different people from different fields, which are connected to our interested area from a different direction to gain a holistic view of the problem area. The total number of respondents for the interviews is nine. All nine interviews help us to understand the actual use of cloud computing architectures in health research collaboration.

\section{TABLE-2: PARTICIPANT JOB ROLES AND REPRESENTATION}

Job role No of participants

Principal Investigators / Project
administrators usually from health /
medical / social sciences backgrounds

2 Cloud specific experts 2

3 Systems Administrators 2

3 Health IT - Data managers/ Database administrators

The selection of different professional interviewees encouraged us to design the questionnaire with different questions focusing on their related area of interest and technologies in used. While constructing the questions for interview, it was kept in the mind that the interviewees who are technical should easily understand the main goal of the questions. To achieve this result we used information technology word instead of Cloud Computing while interviewing the non-technical people. These interviews were conducted for $40-60$ minutes, of course upon consent that the interview can shoot up than expected. The researcher would hold the conversation while taking notes and occasionally asked for missing information. The interviews were audio-recorded and carefully transcribed. To ensure as much accuracy and having the interviewee on point as possible, the audio recordings were listened to again and again and generated transcripts inspected for any missing information or errors.

\section{Data Analysis}

The study's data was analyzed using Glasserian grounded theory approach that involves the use of open coding to help develop concepts / themes and categories through constant comparisons technique. Asserted by [28] study, data analysis is systematic and ought to begin as soon as data is availed, identifying key categories and connecting them. Significant points were identified from the various transcripts of the interviews by organizing them in a tabular form making it possible to distinguish statements from interviewer and responses from interviewees. Identifiers were utilized for each key issue within the transcripts with CSP or HRC/IT, to denote originating participant profile types. For example CSP - Cloud Service Provider or HRC/IT - Health Research Collaborator / Information Technologist (including principal investigator, data managers, and Database administrators). The detail was coded for determining the frequencies of key issues amongst the interviewee responses and concept generation. The key issues upon capture were often italicized for quick reference. More so, the key issues and coding helped in enabling tracking back to originating transcripts. This approach was utilized on all text transcripts of the interview exercises as described by the table that follows.

In table-3, Identifiers are placed on the left of the table with HRC/IT -1 indicating that this key issue was raised from a text transcript of a health research collaboration/IT expert and numbers denote the number of key issue from the same participant profile type, the middle part of contains the key issues while the right part of the table shows the codes of interest. These codes are observed and when similarity is attained among codes through a constant comparison technique, those that are similar are grouped together, resulting into a higher level of regroupings called concepts or high level themes.

Constant comparison of the generated concepts, introduces core categories and later, a theory. The technique is iterative, follows further revisiting of the transcript text of the interviews until when there no more key issues can be identified from the data, the theoretical saturation point, and then concept generation is terminated. Four main thematic categories emerged from the analysis: (a) Reliability Privacy, Confidentiality, integrity; (b) Data Sharing; (c) Innovation Resistance and Organizational Change; (d) Cloud Computing benefits and Opportunities; as also described in findings Section IV. These categories arise from the iterations as issues affecting public health and biomedical health research collaborations based on Cloud Computing. The choice of the categories: - (a) Reliability Privacy, Confidentiality, integrity is because these three issues contribute to the research environment's reliability; (b) Data Sharing- is uniquely observed from interviews with sharing challenges associated to a number issues including institutional sharing policies, governing data laws and the delicate nature of data being handled while the rest (d) Cloud Computing benefits and Opportunities - identified as positive continues to seek that the implementers to ensure a certain level of trust is reached between researchers and their providers.

\section{FINDINGS}

In this section, the research describes the grounded theory of issues affecting health research collaboration based on cloud computing in busy health research environment characterized with south-south and north-south modes of collaborations.

The consequences of the analysis described in the analysis section above form the basis of the contributions of this paper. Preliminarily, the research affirm that while [28]identifies the cloud computing paradigm to be at its infancy stage in the sub-Saharan Africa, its applicability in health care / health research collaboration seems presents the paradigm at its youthful stage. This is true because an inquiry 
into participant knowledge of cloud computing indicated that most of the interviewees seemed to be aware of it with some of the respondents claiming to have often used it and knows confidential. Privacy and confidentiality of patients data has been compromised, one can sell personal information about an individual to third parties.

\section{TABLE-3: ISSUES AND THEIR CODES USED IN THE ANALYSIS}

\begin{tabular}{ll}
\hline \hline IDs (CSP HRC/IT) & Key Issues \\
\hline CSP-1 & "General assumption of trust by health care providers and their \\
& patients that protection is provided by both industry and government \\
& regulations including safe guarding verbal and written interactions \\
& and agreements between all parties regarding patients' personal and \\
& medical data."
\end{tabular}

CSP-2

HRC/IT -1

HRC/IT-2

\begin{abstract}
"Control over proprietary data is lost: - Transition to C storage removes the responsibility of HRCs from supporting and maintaining the software, applications and IT infrastructure that houses the data in the Cloud. Implies data mismanagement, May be an enabler for intrusion by malicious entities"
\end{abstract}

\begin{abstract}
" a cost effective solution that can be easily implemented and accessed by multiple users anywhere without any additional costs such as software licenses, hardware, ICT infrastructure support and maintenance. Its benefits include but not limited to cost reduction, availability and accessibility, scalability and efficiency of the data, with challenges like security and data misuse; confidentiality, integrity, access, power interruptions, unreliable internet."
\end{abstract} "CC challenges existing practices of computing requiring users to
change their attitudes, preferences, and or behaviors."

"Vendors of CC have a deliberate role of ensuring that they explain to their customers about their products and usage procedures.

Details of the products - CC attributes need to be well stipulated to their customers including:

System availability and how it is affected by unreliability of the internet; Data collection, storage and access; Data and Information sharing; CC security; Privacy challenges; Organization change"

\section{Codes}

trust concerns

Inadequate security

Loss of data control

Data ownership

Cost reduction, Availability and Accessibility, Scalability

Data misuse, confidentiality, integrity, access, power interruptions, unreliable internet

Limited Service Level Agreements (SLA) ${ }^{1}$ education,

System unavailability, uneven Data collection, storage and inaccessibility

Heterogeneous data centric insecurity, limited sharing, loss of privacy

\begin{abstract}
it: "I know about cloud computing and I have used cloud application such as Google Apps and Skype", providing also a definition: "Cloud computing is a flexible way to allocate resources out of a pool, enabling to use processing power according to the user needs". Of course those involved technically like Database Managers defined it as: "Cloud computing is a model for enabling convenient, on-demand network access to a shared pool of configurable computing resources (e.g., networks, servers, storage, applications, and services". However, also interview findings also identified that some of the participants have very limited knowledge of cloud computing and some are confused by cloud computing terminology: "My knowledge is very limited and cloud computing terminology seems to be Confusing to me especially on cloud computing architecture..... may be people who deal with data management know better. Ask the data manager who may explain better".
\end{abstract}

\subsection{Data Privacy, Confidentiality and Integrity:}

Privacy: In healthcare research collaboration, patients reveal their health-related information to healthcare professionals in order to diagnose and treat their sickness. As long as the patient makes up his mind to share their data with more than healthcare professionals, their data is no longer private, but
As one of the interviewee puts it that

"When multiple organizations share resources there is a risk of data misuse".

One of IT experts said "Protection of data is the most important challenge in cloud computing. To enhance the security in cloud computing, it is important to provide authentication, authorization and access control for data stored in cloud".

One of the interviewees recommended that "Partners involved in a health research collaboration need to negotiate strong cloud service agreements with detailed provisions relating to security and privacy of patients data in order to fully understand their liabilities and risks".

One of the interviewees who is involved in data management said that: "Patient data could be lost, misused or be used by unauthorized people... Abuse of patient confidentiality carries heavy fines, including significant costs of recovery". A solution to this challenge still resides at the patient data center and organizations should manage patient privacy, he recommended.

Confidentiality: - Most of the interviewees assert that top vulnerabilities are to be checked to ensure that data is protected from any attacks. Almost all interviewees believe that a security test has to be done to protect data from 
malicious user such as Cross-site Scripting, Access Control mechanisms.

"The main question in cloud computing in health research collaboration is, who is the owner of the patients records, the patient himself, the physician or the cloud service provider?",

Two of the interviewees who are IT experts responded. These experts recommend that when data is stored in the remote server, data confidentiality is one of the important requirements. They advised that to maintain confidentiality data understanding and its classification, users should be aware of which data is stored in cloud and its accessibility.

Integrity: - To provide security to the client data, very few clients are used where only limited resources are available. Personal data such as passwords should not be kept by users so that integrity cannot be compromised. One of the interviewee, a principal investigator puts it that:

"In cloud computing, security should be maintained such that data can be only modified by the authorized person. In cloud based environment, data integrity must be robust to avoid the data loss".

Access: "Data access mainly deals with the data security policies. In health research collaborations, the partners are given access to the section of data based on their needs. The same data cannot be accessed by the other people working in the collaboration". One of the Data managers said.

All interviewees in data management said that various encryption techniques and key management mechanisms are used to ensure that data are shared only with the valid users. The key is distributed only to the authorized parties using various key distribution mechanisms.

One of the interviewees, a Data manager said that: "Privileged user access should be provided since access is given through the internet for all cloud users. Data encryption and protection mechanisms can be used to avoid security risk".

\subsection{Data Sharing:}

Data sharing is becoming increasingly important in health research collaboration. It is thus not surprising that more and more healthcare professionals are demanding data sharing capability on their phones, and computers. Data sharing is becoming increasingly prevalent in many Hospitals. These health care centers are now benefitting from data sharing as this provides an efficient and, safer care of patients. There is no need to always repeat medical history of a patient every time a new health professional is consulted which means no more unnecessary tests. Hence, the health professional gets a complete picture of medical history of a patient when using cloud computing. This is evident from the following statements:

"In Health research collaboration, you do not work in isolation. You have to constantly interact with other organizations. A lot of information is sent and received".

One of the interviewees who is a principal investigator responded.
"EMRs are stored in health care providers own databases".

The Interviewees revealed that, the information is moved to the cloud and shared across health research collaborators.

"With explicit customer's consent, some of the patient's information such as Electronic Medical Records (EMRs), can be moved to the cloud and can be shared with hospitals and physicians that are located in different countries".

One of the Doctors who is a Principal investigator further goes on to say that:

"Data distribution services enable the exchange of key health-related data between organizations, such as electronic health records, patient images, and so on".

I asked the interviewees how they share data collected in health research collaborations. All principal investigators said that the key aspect is fast information access to large data. They said that they share information using the internet. One of the PIs explained the possibility to have much higher quality, the possibility of doing knowledge collaboration, take away the negative aspect of having the geographical location as the limitation of the information. The PI who is a medical doctor stated that the affect is patient participation in the care process and his capability to access from home and everywhere. The Data Manager responded that the (access) availability of information to all involved in the research collaboration is important.

\subsection{Innovation Resistance and Organizational change}

The attributes and complexity of cloud computing increase innovation resistance. The innovation itself is a problem for many users. Cloud computing is distinct from other computing products, as it is offered as a computing service via the Internet, benefitting from remote data servers. Almost all the interviewees claim that, the intangible aspect of cloud computing and the fact that it functions within a virtual environment heightens users' uncertainty and discourages its adoption. One of the interviewees a principal investigator said

"Cloud computing is good in health research collaboration BUT If one is using a personal account to store and share files and are the only "access administrator" for those files, what happens if you leave the institution? Will the institution lose access to all data, as well as the ability to manage others' access to those files?"

There exist Innovation challenges requiring healthcare professionals to change their attitudes, preferences, and/or behaviors to adapt to the attributes of using cloud computing in health care.

One of the respondents in interview mentioned knowledge, level of expertise of user's (education of the users) and technology as major challenges in cloud computing so vendor have to explain their customers about their system and its working procedure.

One of the interviewees who is a cloud specific expert said, 
"Adopting cloud computing system for instance at this hospital involves significant modifications to management of healthcare and also to the boundaries in healthcare system. There is resistance and a gap to implement the cloud service in this hospital, due a culture change, work flow change".

He recommended that an efficient training and learning mechanism that will assist healthcare staff to understand the change well, so that staff can merge into the new operational working process without reluctance.

\subsection{Benefits and opportunities of adopting cloud computing}

Clinical benefits: "Medical personnel like doctors can now use applications that were previously not available. For example, the use of digital pathology, managed through cloud services. This means that we can now consult remote experts". One of the interviewees who is a principal investigator with a medical background responded.

She further said that, "these experts can access patient data remotely and on demand through the Internet. Physicians can review the diagnostic results from places of their convenience perhaps determine the way forward for the patient either to discharge immediately or wait".

Collaboration between researchers, physicians and other health professionals becomes a reality, as patient information is centralized and accessible to authorized users. Patient information can be shared between caregivers, regardless of location, allowing for better-informed decisions. This addresses the need for faster screening of patients

Standards: - There are a variety of standards and best practices available which apply to specific aspects of healthcare IT and can be useful when considering the use of specific cloud services. Cloud services which support relevant standards should offer a better level of interoperability:

"Yes, Standards are available. But, there are many standards that apply to different aspects of healthcare

Solutions; we the users should understand these standards and determine whether these cloud services adhere to relevant standards for the capabilities provided".

He further said "... the data and information stored in the providers database server is shared and exchanged to different parties. Lack of standards for policies, interoperability, and transmission methods will hinder the use of cloud computing services in a healthcare environment".

Cloud Opportunities: Health research Collaboration in Cloud computing has a commercial modeled that allows researchers to eradicate or minimize the need to maintain high-cost in-house hardware, software, and network infrastructures. It also reduces and sometimes eliminates the high-cost of recruiting technical professionals to support and operate the in-house infrastructures and IT solutions.
One of the interviewees who is a cloud expert involved in giving technical services to the researchers in the health research collaboration said:

".. A cloud is designed to be flexible and scalable thus allowing users to increase the capacities of their existing system without investing in new infrastructure components. Collecting patients' data in a central location has in many benefits: Patient records will be accessed anywhere and anytime allowing healthcare providers to have a comprehensive view of the patient's history and provide the most suitable treatments accordingly. "

\section{DISCUSSION}

The purpose of this empirical study was to further investigate issues affecting health research collaborations that are supported by cloud computing technologies so that we ground the challenges and aim extensively to suggest innovative and low-cost strategies and tools to realize and scale up adoption and realize the benefits to its implementation.

In this research we found out that while proponents of cloud computing adoption identified platform security and privacy issues, organizational inertia, loss of governance, and uncertain provider's compliance, and fear for data lock-in (immediate and gradual) as challenges, key implementers of Cloud computing based health research collaborations identified that adoption of such technologies is affected negatively also by its reliability including lack of trust by users for privacy, confidentiality, and integrity, inaccessibility dictated by unfairly developed usage Service Level Agreements (SLAs), data sharing inefficiencies and lack of cloud computing knowledge. These alone didn't emanate from the grounded theory use on transcript text but included also limited SLA education, underlying system unavailability, power interruptions, and unreliable internet Its delayed up take is also attached to organizational change and innovation resistance.

With these challenges upfront, user trust, privacy and confidentiality are compromised; deprive efficient and secure and safe patient and research data sharing and access. This not only compromises safety and secure access to patient and research data, but also affects efficient diagnosis since patient medical history cannot be accessed whenever needed, with researchers' belief of Cloud Computing as a virtual environment also amplifies users' uncertainty and discourages its adoption in collaborations.

Increased usage in health research collaboration by these users is attributed to inherent benefits and opportunities of these technologies including enabling of digital pathology, remote and on-demand data access, a business model that allows researchers to minimize the need to maintain high-cost in-house hardware, maintaining technical IT, software, and network infrastructures as identified also in similar adoption related research issues like adoption business enterprises[28] and others.

Extending technologies of scale like cloud computing - blockchain integration into research collaboration whilst observing the inherent issues that may affect efficiency has promise for mending and facilitating a mutual relationship between researchers in developing and developed countries. The integration will re-affirm security and power imbalances amongst researchers since there will 
be equitable access to sharable data and guaranteed availability at all times.

We recommend that research managers and key administrators of these research institutions in order to implement collaborative projects based on Cloud Computing need to ensure increased reliability of the environment and the security of underlying data, implement mechanisms for increased trust. More so, increased Cloud computing education of researchers is required by vendors for their customers including the technologies and their working procedure; the need to managed access and sharing of data, thus enabling data contributors (researchers) to high quality amount of research and EHR data among others.

\section{CONCLUSION}

This paper has explored what users under collaboration: Health IT, Principal Investigators, and Cloud Computing providers observe Cloud Computing use in HRC through an extensive empirical field study, extending our understanding initially supported by literature review. Supported by grounded theory, we have identified critical issues affecting users under health research collaboration using cloud computing. These mainly included Reliability (Data privacy, confidentiality, and integrity); data sharing; organizational change and innovation resistance, negatively delaying the quick adoption.

This paper however also reports that from key users under collaboration that opportunities and benefits of Cloud Computing as identified from a rigorous application of grounded theorem on transcript files as reasons for increased usage. We conclude that the issues affecting HRC using Cloud Computing are double pinned; affecting negatively and positively collaboration prowess.

Ensuring that these challenges are reduced and eliminated through foreseeing a more efficient could environment supporting that attainment of reliability, managed and accessible data sharing, and inclusive SLA and Cloud Computing education among current and prospective researchers. In this paper contributes evidently from a user perspective to Cloud computing literature, generate emerging concepts and attempting to articulate challenges for fast/slow adoption in a busy health research institution in the global south like Uganda.

\section{REFERENCES}

[1] J. W. Lau, E. Lehnert, A. Sethi, R. Malhotra, G. Kaushik, Z. Onder, $e$ al., "The Cancer Genomics Cloud: Collaborative, Reproducible, and Democratized-A New Paradigm in Large-Scale Computational Research," Cancer research, vol. 77, pp. e3-e6, 2017.

[2] P. Mell and T. Grance, "The NIST definition of cloud computing (draft)," NIST special publication, vol. 800, p. 7, 2011.

[3] C. Pino and R. Di Salvo, "A survey of cloud computing architecture and applications in health," in International Conference on Computer Science and Electronics Engineering, 2013.

[4] C. McLeod, "Cloud Computing Service for SMEs Launched in Uganda," ed, 2013.

[5] A. M.-H. Kuo, "Opportunities and challenges of cloud computing to improve health care services," Journal of medical Internet research, vol. 13, 2011.
[6] M. Li, S. Yu, Y. Zheng, K. Ren, and W. Lou, "Scalable and secure sharing of personal health records in cloud computing using attribute-based encryption," IEEE transactions on parallel and distributed systems, vol. 24, pp. 131-143, 2013.

[7] M. P. D. A. Chokshi, and D. P. Kwiatkowski,. (2006) Data sharing and intellectual property in a genomic epidemiology network: policies for large-scale research collaboration,. Bulletin of the World health Organization. 382-387.

[8] C. Olivier, M. R. Hunt, and V. Ridde, "NGO-researcher partnerships in global health research: benefits, challenges, and approaches that promote success," Development in Practice, vol. 26, pp. 444-455, 2016

[9] A. F. Fowler, "Authentic NGDO partnerships in the new policy agenda for international aid: dead end or light ahead?," Development and Change, vol. 29, pp. 137-159, 1998.

[10] R. Zachariah, N. Ford, B. Draguez, O. Yun, and T. Reid, "Conducting operational research within a non governmental organization: the example of Medecins Sans Frontieres," International Health, vol. 2, pp. $1-8,2010$.

[11] A. Costello and A. Zumla, "Moving to research partnerships in developing countries," BMJ: British Medical Journal, vol. 321, p. 827, 2000.

[12] J. Kirschnick, J. M. A. Calero, L. Wilcock, and N. Edwards, "Toward an architecture for the automated provisioning of cloud services," IEEE Communications Magazine, vol. 48, pp. 124-131, 2010.

[13] M. Li, S. Yu, K. Ren, and W. Lou, "Securing Personal Health Records in Cloud Computing: Patient-Centric and Fine-Grained Data Access Control in Multi-owner Settings," in SecureComm, 2010, pp. 89-106.

[14] M.-G. Avram, "Advantages and challenges of adopting cloud computing from an enterprise perspective," Procedia Technology, vol. 12 , pp. 529-534, 2014

[15] R. Buyya, C. S. Yeo, S. Venugopal, J. Broberg, and I. Brandic, "Cloud computing and emerging IT platforms: Vision, hype, and reality for delivering computing as the 5th utility," Future Generation computer systems, vol. 25, pp. 599-616, 2009.

[16] I. Budin-Ljøsne, P. Burton, J. Isaeva, A. Gaye, A. Turner, M. J. Murtagh, et al., "DataSHIELD: an ethically robust solution to multiple-site individual-level data analysis," Public health genomics, vol. 18, pp. 87-96, 2015.

[17] L. A. B. Silva, C. Costa, A. Silva, and J. L. Oliveira, "A PACS Gateway to the Cloud," in Information Systems and Technologies (CISTI), 2011 6th Iberian Conference on, 2011, pp. 1-6.

[18] C. T. Yang, Chen, L.T., Chou, W.L. and Wang, K.C, "Implementation of a medical image file accessing system on cloud computing. In Computational Science and Engineering (CSE),," in IEEE 13th International Conference on (pp. ). IEEE., December, 2010, pp. 321-326.

[19] M. J. Khoury, T. K. Lam, J. P. Ioannidis, P. Hartge, M. R. Spitz, J. E. Buring, et al., "Transforming epidemiology for 21st century medicine and public health," Cancer Epidemiology and Prevention Biomarkers, $\mathrm{p}$ cebp. 0146.2013, 2013.

[20] Y. Demchenko, P. Grosso, C. De Laat, and P. Membrey, "Addressing big data issues in scientific data infrastructure," in Collaboration Technologies and Systems (CTS), 2013 International Conference on, 2013, pp. 48-55.

[21] Y. Demchenko, Z. Zhao, P. Grosso, A. Wibisono, and C. De Laat, "Addressing big data challenges for scientific data infrastructure," in Cloud Computing Technology and Science (CloudCom), 2012 IEEE 4th International Conference on, 2012, pp. 614-617.

[22] B. Glaser and A. Strauss, "Grounded theory: The discovery of grounded theory," Sociology The Journal Of The British Sociological Association, vol. 12, pp. 27-49, 1967.

[23] W. J. Orlikowski. (1993) CASE tools as organizational change: Investigating incremental and radical changes in systems development. MIS quarterly. 309-340.

[24] E. Dunican, "Initial experiences of using grounded theory research in computer programming education.," in Proceedings of the 18th Workshop of the Psychology of Programming Interest Group, 2006, pp 183-197.

[25] E. Dunican, "Initial experiences of using grounded theory research in computer programming education," in Proceedings of the 18th Workshop of the Psychology of Programming Interest Group, 2006, pp. 183-197.

[26] L. K. Nelson. (2017) Computational Grounded Theory: A Methodological Framework. Sociological Methods \& Research. 0049124117729703

[27] B. a. C. DiCicco- Bloom, B.F., "The qualitative research interview. ," Medical education, vol. 40, pp. 314-321, 2006.

[28] S. D. Seifu, Dahiru, A.A., Bass, J.M. and Allison, I.K., 2017, "Cloud- Computing: Adoption Issues for Ethiopian Public and Private 
Robert Mugonza is a lecturer in the department of Computer Science, Faculty of Computing \& Informatics, Mbarara University of Science and Technology. He holds a MSc in Advanced Computer Science from University of Leeds and a Bachelor of Computer Science from Mbarara University of Science and Technology.

Robert is a member of the IEEE Computer Society and IEEE Cloud Computing. His research areas of interest are in Internet of Things, Cloud Computing, Modeling, Design and Analysis of Systems, and Software Engineering.

Dr. Annabella Habinka Basaza-Ejiri is the Academic Registrar of St. Augustine International University and Associate Professor in the College of Computing and Engineering. She holds a $\mathrm{PhD}$ in Information Systems from Groningen University in the Netherlands, MPhil. S\& T from Stellenbosch University in South Africa, a Master's of Science degree in Information System from Makerere University and a Bachelor of Computer Science from Mbarara University of Science and Technology.

She is an associate member of the Association for Information Systems and a member for IEEE computer Society with areas in Software Engineering; Cloud computing, Information Systems and Decision Support. 